\title{
Factores de riesgo de tromboembolismo venoso en la población hondureña
}

Milena Vanegas ${ }^{1}$

Ingrid Urbina ${ }^{2}$

\section{RESUMEN}

Las mutaciones factor V Leiden y protrombina G20210A (FII), las deficiencias de la antitrombina, proteínas $\mathrm{C}$ y $\mathrm{S}$ y el incremento de procoagulantes como el fibrinógeno constituyen factores de riesgo relacionados con el tromboembolismo venoso, una entidad clínica de alta incidencia y morbilidad en el mundo entero. El objetivo del estudio fue estimar la prevalencia de estos factores de riesgo asociados con trombofilia en una muestra de población saludable de Honduras. Se procesaron 93 muestras de participantes de ambos sexos entre las edades de 17 a 70 años, sin antecedentes de eventos trombóticos ni padecimientos agudos o crónicos como diabetes, lupus eritematoso sistémico, cáncer, trastornos mieloproliferativos, patologías hepáticas o renales.

El estudio fue aprobado por el Comité de Ética del Instituto Hondureño de Seguridad Social y la información de cada participante se obtuvo a través de un consentimiento informado. Las proteínas de la coagulación se determinaron por métodos estándar basados en coágulo y turbidimétrico (stago human) y los polimorfismos alélicos de factor $V$ Leiden y protrombina G20201A se determinaron por método de reacción de polimerasa en cadena (PCR) en tiempo real (LightCycler 2.0, Roche).

Los resultados del estudio revelan niveles moderadamente disminuidos de la actividad de antitrombina, proteína C y $S$ en un $6.4,10.7$ y $11.8 \%$ de la muestra, respectivamente. En general, las frecuencias encontradas son más altas que las reportadas en la población general en la literatura científica, la cual no es mayor a $2 \%$.

1 Profesora universitaria beneficiaria de una beca sustantiva de la DICYP. Directora Escuela de Microbiología, Facultad de Ciencias, UNAH: milenavanegas@unah.edu.hn

2 Gerente de Gestión Hospitalaria de Medicina Interna, Hospital de Especialidades, IHSS: hollmann7@ hotmail.com 
Un $5.4 \%$ de la muestra reveló niveles levemente aumentados de fibrinógeno. En relación a los polimorfismos genéticos, la frecuencia de la mutación de la protrombina G20210A fue de $2.15 \%$ en forma heterocigota, similar a la reportada en el resto del mundo, y no se detectó ningún caso de factor $\mathrm{V}$ Leiden, pese a ser el factor genético más frecuentemente encontrado en caucásicos europeos y estadounidenses (5-15\%) y en varios países de Latinoamérica (1.6 \% a 5,1\%).

No se observó más de una alteración de los factores de riesgo en los participantes. Los resultados del estudio, aunque no generalizables por el tamaño de la muestra, revelan alteraciones de la hemostasia en la población estudiada. La evaluación del perfil trombofílico en el laboratorio de coagulación y hemostasia sería valioso en la prevención del tromboembolismo venoso en pacientes seleccionados expuestos a situaciones de riesgo, por lo que sería importante desarrollar en el país estos estudios. Por primera vez se describe la mutación de la protrombina G20210A en la población hondureña.

Palabras clave: tromboembolismo venoso, factores de riesgo, trombofilia, mutación factor V Leiden, mutación G20210A de la protrombina, proteína S, proteína C, antitrombina, fibrinógeno.

\section{ABSTRACT}

Factor V Leiden and Prothrombin G20210A (FII) mutations, Antithrombin, Protein C and $S$ deficiencies, and high levels of Fibrinogen are related genetic risk factors for venous thromboembolism which exhibits high incidence and morbidity worldwide. The objective of this study was to determine the prevalence of these risk factors in healthy individuals of Honduras. 93 samples were collected from both gender participants between 17 to 70 years old, with no previous history of venous embolism, acute or chronic diseases such as diabetes, systemic lupus erythematosus, cancer, mieloproliferative disorders, liver and renal diseases. The study was approved by the Ethical Review Board of the Social Security National Institute and the information was obtained through an Informed Consent Form. Clot based and turbidimetric methods were used to measure the control proteins (Stago, Human) and real time polymerase chain reaction (RT-PCR, LightCycler 2.0, Roche) was carried out to screen Factor V Leiden and Prothrombin G20210A. The frequencies of Antithrombin, Protein C y S decreased levels were 6.4, 10.7 and $11.8 \%$, respectively. In general, our 
data was higher than the reported in the general population, with levels less than $2 \%$. Fibrinogen levels were found increased in $5.4 \%$ of the sample. In regard the allelic polymorphism, Prothrombin G20210A frequency was $2.15 \%$ with heterozygous form, similar to the reported worldwide; no Factor $V$ Leiden mutation was detected even if its average prevalence in the Western population is $10-15 \%$ and in Latin-Americans is $1.6-5.1 \%$. No more than one disorder was observed in the subjects of the study. Our data is not generalizable due to the size of the sample but it reveals hemostasis unbalance in healthy subjects. Thrombophilic screening in the hemostasis laboratory may be valuable in selected patients exposed to risky procedures or medications, to prevent venous embolism with anticoagulation therapy. It would be important to develop these studies in the country to contribute for the venous embolism prevention and management. Prothrombin G20210A mutation is first time reported in the Honduran population.

Keywords: venous thromboembolism, risk factors, thrombophilia, factor $V$ Leiden mutation, prothrombin G20210A mutation, protein S, protein C, antithrombin, fibrinogen. 


\section{INTRODUCCIÓN}

El riesgo de tromboembolismo venoso ha sido asociado a condiciones genéticas y adquiridas, recibiendo mucha atención científica en las últimas décadas por su alta incidencia y morbilidad en el mundo, ya que al menos el $30 \%$ de la población mundial fallece por una condición trombótica arterial o venosa y en un $25 \%$ de los casos el evento inicial es fatal (Arruda, Annichino Bizzacchi y Costa, 1995; Dutta, 2009).

Muchas de las trombosis fatales no son diagnosticadas antes de una autopsia. Los principales factores de riesgo genéticos que han sido identificados son las deficiencias de antitrombina, proteína $C$ y proteína $S$. Recientemente, con el desarrollo de las técnicas moleculares, se han identificado varios polimorfismos genéticos que predisponen a la trombosis, siendo descritos dos de ellos como los de mayor prevalencia las mutaciones factor $V$ Leiden y la protrombina G20210A (Fritsma, 2012; Herrmann y otros, 1997; Middeldorp, 2011; Miranda- Vilela, 2012).

La mutación factor $\mathrm{V}$ Leiden confiere resistencia a la proteína $\mathrm{C}$ activada incrementando el riesgo de trombosis en portadores heterocigotos 3-7 veces y en homocigotos 80 veces (Dutta, 2009; Herrmann y otros, 1997). La mutación de la protrombina G20210A se asocia a un nivel plasmático más alto de protrombina e incrementa 1.6-11.5 veces el riesgo de trombosis en un individuo heterocigoto (Dutta, 2009; Herrmann y otros, 1997; Miranda- Vilela, 2012).

El producto de los estudios científicos ha contribuido para entender mejor la patogénesis de la enfermedad, revolucionando el pronóstico y manejo de esta condición que amenaza la vida de un individuo por la complicación asociada al tromboembolismo pulmonar (Dutta, 2009).

Este es el primer estudio en el país tendiente a estimar la prevalencia de algunos factores de riesgo asociados al tromboembolismo venoso en una muestra de la población hondureña y contribuir a generar conocimientos científicos en la patogénesis del evento trombótico, influenciar sobre la duración de la terapia después del episodio, individualizar el riesgo y facilitar lineamientos para la prevención de la trombosis, especialmente cuando los individuos se encuentren en situaciones que incrementen el riesgo, tales como embarazo, cirugías, inmovilización, terapia de reemplazo hormonal, entre otros (Ornstein, 2003; Pereira y otros, 1996; Ruiz-Arguelles, Garces-Eisele y Reyes-Nuñez, 2001). 
Se investigaron los niveles de actividad de proteínas $S$ y $C$, antitrombina, concentración de fibrinógeno, y los polimorfismos alélicos de factor $V$ Leiden y protrombina G20210A. El objetivo fue estimar la prevalencia de algunos factores de riesgo asociados con la trombofilia en una muestra de población sana de Honduras.

\section{MATERIALES Y MÉTODOS}

Durante 10 meses se captaron 93 participantes sanos, de ambos sexos, que laboran en la Escuela de Microbiología, UNAH, y en el Laboratorio Clínico del Hospital Escuela Universitario de Tegucigalpa, comprendidos entre las edades de 17 y 70 años, quienes voluntariamente participaron en el estudio. $46 \%$ de los participantes fueron de sexo masculino y $54 \%$ sexo femenino, sin antecedentes de haber padecido algún evento trombótico, enfermedades agudas o crónicas tales como diabetes mellitus, glomerulopatías, hepatopatías, síndromes mieloproliferativos, lupus eritematoso sistémico y cáncer; además de ausencia de embarazo, fracturas, reposo prolongado, uso de anticonceptivos orales, terapia de reemplazo hormonal y cirugía reciente.

Se determinó la concentración de fibrinógeno por el método convencional de Clauss (Human) y los niveles de actividad de los inhibidores que se analizaron fueron las proteínas $\mathrm{S}$ y $\mathrm{C}$ por métodos basados en coágulo (Stago), el nivel de actividad de antitrombina por método turbidimétrico (Stago). Los polimorfismos alélicos de factor $\mathrm{V}$ Leiden y protrombina G20201A se determinaron por método de reacción de polimerasa en cadena mediante sondas FRET en tiempo real (Light Cycler 2.0 de Roche). Se aplicó el cálculo matemático de porcentaje para estimar la frecuencia de los factores de riesgo estudiados. El estudio fue aprobado por el Comité de Ética del Instituto Hondureño de Seguridad Social y a cada participante se le aplicó un instrumento y se obtuvo consentimiento informado.

\section{RESULTADOS}

Los resultados de la investigación revelan alteraciones de los niveles de los factores de riesgo estudiados, con niveles de actividad leve a moderadamente disminuidos en los anticoagulantes naturales, alcanzando el mayor porcentaje de disminución la proteína S con un $11.8 \%$. El procoagulante, fibrinógeno, se encontró un $5.4 \%$ con niveles leve a moderadamente aumentados (ver tablas 1 y 2 ). 
Con relación a los polimorfismos alélicos, se detectó la mutación de la protrombina G20210A con una frecuencia de $2.15 \%$ y ningún caso de la mutación de factor $V$ Leiden en la muestra del estudio. Por primera vez se describe esta mutación en la población hondureña (ver tabla 3). Se obtuvo consentimiento de los familiares en primer grado de consanguinidad de uno de los participantes del estudio detectado con la mutación de la protrombina G20210A para el estudio de la mutación, encontrándose el padre del participante positivo por la mutación (ver figura 1).

Tabla 1. Resultados disminuidos del nivel de actividad de inhibidores de la coagulación $(n=93)$

\begin{tabular}{l|c|c|}
\hline Inhibidor & $\begin{array}{l}\text { No. muestras (\%) con rango } \\
\text { actividad disminuido }\end{array}$ & Rango de actividad disminuido \\
\hline $\begin{array}{l}\text { Antitrombina } \\
\text { valor de referencia 78-126 \% }\end{array}$ & $6(6.4 \%)$ & $56-77 \%$ \\
\hline $\begin{array}{l}\text { Proteína C disminuidos } \\
\text { valor de referencia 70-140 \% }\end{array}$ & $10(10.7 \%)$ & $31-64 \%$ \\
\hline $\begin{array}{l}\text { Proteína S disminuidos } \\
\text { valor de referencia 65-140 \% }\end{array}$ & $11(11.8 \%)$ & $39 \%$ \\
\hline
\end{tabular}

Tabla 2. Resultados de fibrinógeno

\begin{tabular}{|l|c|c|}
\hline Inhibidor & No. muestras & $\%$ \\
\hline $\begin{array}{l}\text { Niveles de fibrinógeno en el intervalo } \\
\text { de referencia (220-498 mg/dL) }\end{array}$ & 88 & 94.6 \\
$\begin{array}{l}\text { Niveles aumentados de fibrinógeno } \\
\text { (en el rango } 499-582 \mathrm{mg} / \mathrm{dL} \text { ) }\end{array}$ & 5 & 5.4 \\
\hline
\end{tabular}

Tabla 3. Resultados de las pruebas de los polimorfismos alélicos protrombina G20210A y factor V Leiden

\begin{tabular}{|l|c|c|c|}
\hline & & \multicolumn{2}{|c|}{ Positivos } \\
\hline Polimorfismos alélicos & N & No. & $\%$ \\
\hline Protrombina G20210A & 93 & 2 & 2.15 \\
\hline Factor V Leiden & 93 & 0 & 0 \\
\hline
\end{tabular}


Figura 1. Resultados de pesquisa de la mutación de la protrombina G20120A en familiares de primer grado de un participante positivo*

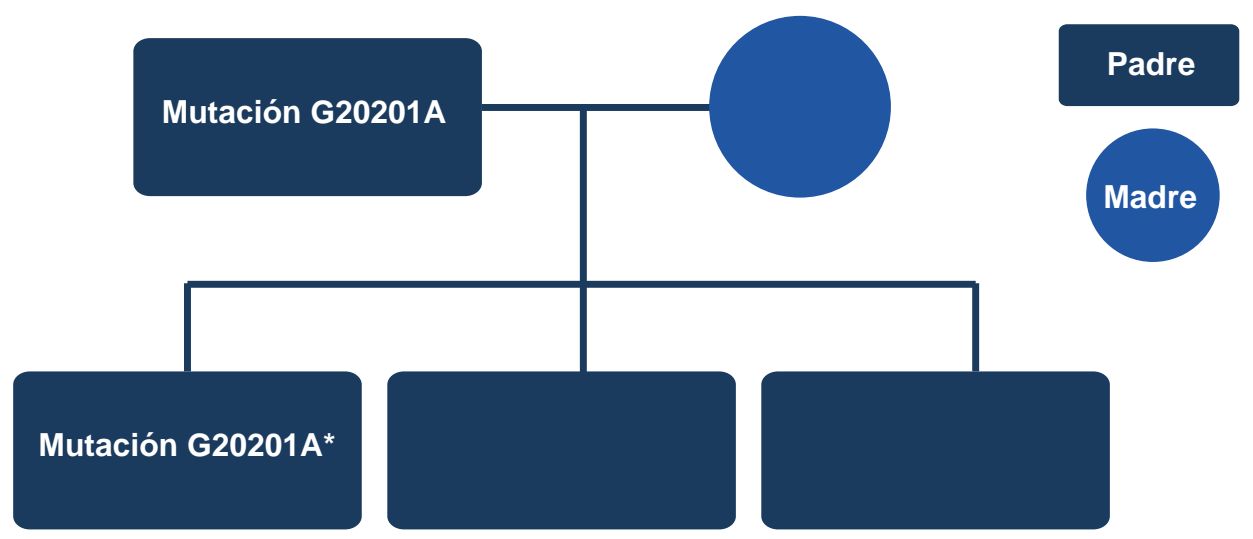

\section{DISCUSIÓN}

Las deficiencias de las principales proteínas anticoagulantes naturales como la antitrombina, proteínas $\mathrm{C}$ y $\mathrm{S}$, se han establecido plenamente desde hace mucho tiempo como factores de riesgo para el tromboembolismo venoso, representando en la población general al menos un $2 \%$ según la literatura científica (Dutta, 2009; Herrmann y otros, 1997; Middeldorp, 2011). Nuestros hallazgos reportan una mayor frecuencia en un $6.4,10.7$ y $11.8 \%$, respectivamente en la muestra estudiada.

El rango de actividad obtenido de estas proteínas responde al fenotipo de individuos heterocigotos quienes no desarrollan un evento trombótico, a menos que se expongan a otras condiciones de riesgo, entre ellas, cirugías, terapia hormonal, malignidades y otros (Herrmann y otros, 1997). El fenotipo heterocigoto se considera incompatible con la vida y los individuos desarrollan trombosis a edad del nacimiento usualmente (Dutta, 2009; Miranda- Vilela, 2012; Middeldorp, 2011; Tripodi, 2001).

La deficiencia de antitrombina detectada es tipo I o cuantitativa, asociada a la producción reducida de la proteína y constituye el $90 \%$ de los casos reportados en la población general. Los valores disminuidos de la actividad se encontraron en el rango de 56 al 77 \% (intervalo de referencia 78-126 \%) (Dutta, 2009; Salomon, Steinberg y Zivelin, 1999). En relación a proteínas C y S, los participantes mostraron niveles de actividad moderadamente disminuidos en un $10.7 \%$ y $11.8 \%$, respectivamente, los 
cuales se relacionan con una vida normal en los individuos, pero con una mayor vulnerabilidad a la trombosis dado el rol vital que juegan en la inactivación de factores activados (ver tabla 1).

Fue sorprendente encontrar una tasa superior a la reportada en estudios similares y es recomendable realizar un estudio para establecer los intervalos de referencia en nuestra población, pues el utilizado para todas las pruebas son los sugeridos por el fabricante de los reactivos utilizados. En el estudio no se descarta la posibilidad de alguna inactivación de las proteínas, dada la vida media corta que poseen de 6 horas (proteínas C y S) y 72 horas (antitrombina). Estos inhibidores son proteasas potencialmente vulnerables a artefactos, entre ellos, altas concentraciones de factor VIII de la coagulación, activación de factor VII por tiempo prolongado de congelación, temperatura ambiental, por lo que el plasma debe conservarse de manera inmediata a menos $20^{\circ} \mathrm{C}$, sino se procede a su procesamiento el mismo día de la toma de la muestra. Durante el estudio hubo algunas interrupciones de energía eléctrica que pudieran haber interferido en los resultados, lo cual puede producir valores subestimados (Middeldorp, 2011; Ruiz-Arguelles, Garces-Eisele y Reyes-Nuñez, 2001; Salomon, Steinberg y Zivelin, 1999).

Se encontró en un $5 \%$ de la población estudiada niveles de fibrinógeno mayores a $500 \mathrm{mg} / \mathrm{dL}$. El fibrinógeno fue el único procoagulante que se incluyó en el estudio y se ha asociado a trombosis venosa con un riesgo relativo (RR) de 4. Estos datos en la población hondureña son más altos que los reportados en otras investigaciones similares (ver tabla 2) (Dutta, 2009; Middeldorp, 2011; Tripodi, 2001).

Se detectaron 2 casos positivos ( $2.15 \%$ ) con mutación heterocigota de protrombina G20210A y ningún caso de la mutación de factor $V$ Leiden. En la pesquisa a los familiares de primer grado de consanguinidad (padres y tres hermanos) de un participante portador heterocigoto de la mutación de protrombina G20210A, se encontró positivo el padre, quien no ha sufrido ningún evento de tromboembolismo venoso (ver gráfico 1).

La frecuencia de la mutación de la protrombina G20210A de $2.15 \%$ es similar a la reportada en población europea, estadounidense y latinoamericana por la comunidad científica. (Fritsma, 2012; Herrmann y otros, 1997; Middeldorp, 2011). En cuanto al factor $\mathrm{V}$ Leiden, pese a que constituye el factor de riesgo genético más frecuentemente encontrado en los caucásicos europeos y estadounidenses con una frecuencia 5-15\%; (Dutta, 2009; Middeldorp, 2011; Ornstein, 2003); en Latinoamérica con una frecuencia de $1.6 \%, 5,1 \%$ y $3,8 \%$ en Venezuela, Argentina y Costa Rica, 
respectivamente; en Brasil y Chile en un $2 \%$, pero en nuestro estudio no se detectó ningún caso en la muestra estudiada (Dutta, 2009; Pereira, y otros, 1996; Ruiz-Arguelles, Garces-Eisele y Reyes-Nuñez, 2001; Salomon, Steinberg y Zivelin, 1999; Tripodi, 2001; Organization, 2014). No se observó más de una alteración de los factores de riesgo en los participantes.

\section{CONCLUSIONES}

Los resultados de la investigación revelan una disminución de leve a moderada de los inhibidores de la coagulación, antitrombina, proteína C y S, en la población estudiada, sin antecedentes de evento trombótico venoso. También se encontró incremento en los niveles de fibrinógeno en un $5 \%$. Los datos obtenidos en la población hondureña son más altos que los reportados en otras investigaciones similares (Dutta, 2009; Ornstein, 2003; Organization, 2014).

La frecuencia encontrada de la mutación de la protrombina G20210A de $2.15 \%$, no varía respecto a la reportada por la mayoría en otros países europeos, latinoamericanos y Estados Unidos de América. (Fritsma, 2012; Middeldorp, 2011). Este es el primer estudio en Honduras en reportar la mutación del polimorfismo alélico de protrombina G20210A. Sin embargo, nuestros resultados difieren con los reportes de investigadores de esos países en relación a la mutación factor $V$ Leiden, la cual no fue detectada y es descrita como el factor de riesgo genético más frecuentemente encontrado en los caucásicos europeos y estadounidenses, con una frecuencia 5-15 \% (Dutta, 2009; Middeldorp, 2011). Quizá los hallazgos en la población hondureña son más cercanos a los descritos por Ruiz Arguelles y colaboradores (2001) en la población mestiza mexicana con una tasa más alta de protrombina G201201A que $F$ $\checkmark$ Leiden.

Un estudio con mayor muestra es recomendable para establecer la frecuencia de factor $V$ Leiden en el país. Aunque en nuestro estudio no se detectó ningún caso, un laboratorio clínico privado (único en el país en realizar la prueba) ha reportado a la fecha 3 casos positivos de un total de 77 pacientes, en un periodo aproximado de tres años, evidenciando la presencia de la mutación en Honduras. Los casos corresponden a pacientes seleccionados con patología tromboembólica en curso 0 bajo sospecha.

La detección de la mutación de la protrombina G20210A en el padre de un 
participante positivo, con 78 años de edad y sin historia médica de evento trombótico, reafirma la opinión de muchos autores y del equipo de esta investigación, que es poco justificado el estudio de factores de riesgo hereditarios en nuestro medio como rutina del estudio de trombofilia.

Dado que en el país se empiezan a realizar estudios genéticos, es importante apuntar que una buena práctica clínica demanda la consejería genética, por lo que el personal médico deberá tener la capacidad de proveer una explicación clara y precisa a individuos portadores de mutaciones, que no subestime o sobreestime la presencia de una mutación.

Los resultados de la investigación no son generalizables por el pequeño tamaño de la muestra, sin embargo, proveen evidencia importante de alteraciones en niveles de factores protrombóticos y anticoagulantes en la población hondureña que afectan la hemostasia y que pueden ser investigados en el laboratorio de coagulación. En individuos seleccionados con historia familiar de trombosis y que se someten a procedimientos que potencian un mayor riesgo de un evento trombótico, el perfil laboratorial de factores de riesgo podría ser valioso para aplicar una terapia preventiva con anticoagulación farmacológica.

En nuestro medio es común que la enfermedad autoinmune ocupe la principal sospecha diagnóstica en la etiología de la trombosis venosa, nuestros hallazgos sugieren que alteraciones de las proteínas de control de la coagulación en individuos sin otras enfermedades de base o mutaciones, podrían ser consideradas en la etiopatogénesis de la enfermedad permitiendo mejorar el abordaje clínico (Herrmann y otros, 1997; Miranda- Vilela, 2012).

El rol del laboratorio de hemostasia y coagulación se redefine con la caracterización molecular de las mutaciones implicadas en la etiología de la trombosis venosa y contribuye al manejo clínico y es importante potenciar su desarrollo en el país.

\section{AGRADECIMIENTO}

Este estudio fue realizado con el valioso apoyo de la Dirección de Investigación Científica y Posgrado de la UNAH, mediante una beca sustantiva de investigación. Se agradece a las doctoras Blanca Alejandra Hernández e Iveth Olinda Núñez, por su colaboración en el estudio. 


\section{BIBLIOGRAFÍA}

Arruda, V.; Annichino Bizzacchi, J. M. y Costa, F. Y. (1995). Factor V Leiden (FVQ $506)$ is common in a Brazilian population. Am J Hematol, 242- 243.

Dutta, T. Y. (2009). Venous thromboembolism: The intricacies. Journal of Postgraduate Medicine, 55 - 64.

Fritsma, G. (2012). Hematology, Clinical Principles and Applications. St. Louis, Missouri: Elsevier Saunders.

Herrmann, F.; Koesling, M.; Schorder, W.; Altman, R.; Jiménez Bonilla, R. y Lopaciuk, S. E. (1997). Prevalece of factor V Leiden mutation in various populations. Genet Epidemiol, 403- 411.

Middeldorp, S. (2011). Evidence-based approach to thrombophilia testing. Throm Thrombolysis, 275-281.

Miranda- Vilela, A. (2012). Role of polymorphisms in Factor V (FV Leiden), Prothrombin, Plasminogen activator inhibitor type-1 (PAI-1), Methylenetetrahydrofolate reductase (MTHFR) and cystathionine B- synthase (CBS) genes as risk factors for thrombophilias. Mini Rev Med Chem, 75- 90.

Ornstein, D. L. (2003). Factor V Leiden. Circulation, e94-e97.

Pereira, J.; Quiroga, T.; Goycoolea, M.; Muñoz, B.; Hidalgo, P. y Kaltwasser, G. E. (1996). Activated C protein resistance: Laboratory study and prevalence of the defect in the Chilean population. Rev Med Chile, 663-668.

Ruiz-Arguelles, G.; Garces-Eisele, J. y Reyes-Nuñez, V. (2001). Primary Thrombophilia in México, Factor V G1691A (Leiden), Prothrombin G20210A, and Methylenetetrahydrofolate Reductase C677T. Polymorphism in Thrombophilia Mexican Mestizos. American Journal of Hematology, 28-31.

Salomon, O.; Steinberg, D. y Zivelin, A. E. (1999). Single and Combined Prothrombotic Factors in Patients With Idipathic Venous Thromboembolism. Prevalence and Risk Assessment. Arterioscler Thromb Vasc Biol, 511-518.

Tripodi, A. Y. (2001). Laboratory Investigation of Thrombophilia. Clinical Chemistry, 1597-1606. 